\title{
FORUM
}

\section{What an Inertial Navigator Consists of}

\author{
Frank Coffman Bell
}

I. Mr. J. G. CARr and SQ.-Leader D. Scotr, R.A.F., have devoted sections two, three and four of a total of $2 \mathrm{I}$, and a few other remarks here and there in their excellent paper, 'The testing of airborne inertial navigation systems' (Journal, 20, 405), to the above title as an exposition 'first necessary' to their main purpose, limiting themselves to 'the types of system of which we have practical experience'. They do not otherwise identify these types explicitly, but do include them among the 'many ways of mechanizing this concept', viz., 'The feature which is common to all inertial navigators is the measurement of acceleration of the vehicle by measuring [and in effect they add also "or metering'] the force required to constrain a proof-mass carried in the vehicle to move with it,' and from this deducing velocity and position of the vehicle. This statement seems well put to me and quite sound, but in the fourth paragraph of Section 4, which includes 'Fig. I. A Schuler tuned inertial navigator', they imply that at least their types, if not all inertial navigators, are based also on 'Schuler tuning [so named] after the discoverer of the principle', and here I do not follow them. (I note in passing that the by-line of Schuler's 1923 paper, Physik. Zeitschr. XXIV, 344-50, spells his name without the umlaut used consistently by them.*) The explanation given by them seems more or less to conform to a standard formula used by all public-spirited writers who touch on the subject of inertial navigation and are anxious to make sure that readers have at least that degree of understanding which is possible for the layman, it being assumed always that this degree is low and does not justify the supposed labour and space of an attempt at undue accuracy or precision in the explanation. And so, of course, the following two criticisms of their explanation do not have to be regarded too narrowly as criticisms of them.

2. There is not anywhere in Schuler's 1923 paper any justification for connecting his name or his principle with inertial navigation. To support this contention, I appeal to Schuler's paper itself, and submit here a complete accounting for the contents of its 37 paragraphs which could be in any way significant to this contention. I do not think that anyone can produce citations of the German language of Schuler which will amount to such justification on any argument, or locate in this accounting where such a citation might occur:

Title: Disturbance of Pendulum- and Top-Apparatuses by Acceleration of the Vehicle.

Pars. 1-5: Historical comments and references. Statements of purpose: to show how, by an 'almost trivial' idea, such disturbances could be avoided, and with a bow in passing to the notion that gravity and acceleration cannot be separated [-Which separation, nevertheless, his devices are designed to achieve.]

* This was, I rather think and regret to say, an editorial amendment. Ed. 
Par. 6: String pendulum of Earth's radius' length, direction and position.

Pars. 7-12: The equivalent and equally impractical compound pendulum. Par. 13: Assertion of the only way, the pendulous top.

Pars. 14-23: Pendulous top as artificial horizon.

Pars. 24-31: Latitude-dependent 'steaming error' of the Anschütz-Kaempfe gyro-compass, and success in controlling the oscillation due to this error by pre-set, latitude-dependent tuning. Contrasting failure of attempts to reduce to practice the top artificial horizon.

Por. 32: A hypothetical and impossible absolute system. By contrast, the only possible relative system-the top horizon as artificial horizon for celestial navigation.

Par. 33: Recommendation: find a way to make the top horizon good enough for celestial navigation, and quickly.

Pars. 34-35: Schuler's own statement of his principle [To the extent that this statement is sound, it is identical with d'Alembert's solution for the problem of the compound pendulum moving in the circumference of a circle and always coincident in direction with the local radius ('Traité de dynamique', 2nd ed., Paris, Davif, $175^{8}$.)].

Par. 36: Discussion of his table of fifteen principal equations--five each for compound pendulum, pendulous top and gyro-compass.

Par. 37: A pleasant comment on the human condition, in particular serendipity.

On tuning: Schuler uses the word 'abstimmen', or 'to tune', three times, twice referring to the pendulous top horizon, pars. 13 and $2 \mathrm{I}$, and once referring to the 'steaming error' oscillation of the Anschütz-Kaempfe gyro-compass, par. 31 .

On the $84^{m}$ period: Schuler gives no references, but by no means implies that he is 'the discoverer' of this 'almost trivial' idea. [An explicit reckoning of this period was given by Ritter in 1873 (Lehrbuch der Analytischen Mechanik, Baumgärtner's, Leipzig, Ist ed., I873, 2nd ed., I883). There probably are earlier published explicit reckonings. Newton appears to have been the first to analyse this oscillation, in 'Principia', Book I, Section X, but he did not give an explicit reckoning of the 84-minute period.]

On accelerometers: Schuler explicitly rules out integrating accelerometers as a necessary element in Schulerian navigation, par. 32.

3. The discussion in Section 4, paragraph 4, using Fig. I, relates in part to Schulerian navigation, and in the remainder to inertial navigation, although Fig. I itself has no counterpart in Schuler's paper and seems perfectly adequate to me as a model of inertial navigation, though not at all appropriate as a model of Schulerian navigation. There would be no harm in this if, as assumed by Carr and Scott explicitly, inertial navigation and Schulerian navigation were one and the same. As I do not follow them in this assumption, I find their discussion confused and unclear in both parts, even though many (if not perhaps all) of their statements taken alone are separately right if the right connection be understood. Now consider the system of Fig. I with vertical gyro and accelerometer cluster, the 'platform', motionless in circumferential position at all times, and level in local rotation when released. The system, though free initially from any real physical error, may have, say, an initial velocity error rightward (interpretable as a 'feedback loop scale factor' error or other 'disturbance' of the 'loop'). The 
computer will then have the illusion that the platform is in circumferential motion to the right, and the computer consequently calls for a clockwise rotation of the gyro, so that the platform actually does physically rotate clockwise, so tilting the accelerometer, but without the circumferential motion rightward assumed by the deluded computer. The position and rate pick-offs on the proof mass then call for a leftward real pressure to maintain the proof mass in null position, by balancing the component of gravity which is due to the real physical tilt of the accelerometer, and is really acting on the proof mass. Thus the accelerometer delivers to the computer a signal which the computer must interpret as that of a leftward tangential acceleration in circumferential motion, and the computer's indication of position, $\theta$, obeys the law, if $m$ be the proof mass,

$$
\frac{m d^{2}(R \theta)}{d t^{2}}+\frac{m g}{R}(R \theta)=0^{*}
$$

which reduces to

$$
\frac{d^{2} \theta}{d t^{2}}+\frac{g}{R} \theta=0
$$

and which has the solution $\theta \equiv 0$, to be sure, but others lso. The platform attitude really and physically oscillates in local rotation about the level according to the same law with $\theta$ properly interpreted as the local angular measure of the platform attitude now. Therefore, in both cases the period is exact and tautochronous with respect to amplitude, and is given by

$$
T=2 \pi \sqrt{\frac{R}{g}} \approx 84^{\mathrm{m}}
$$

no matter what the amplitude. A Schuler pendulum does not have this property, except in the limit as the amplitude vanishes, and equations ( 1 ) and (2) above occur in his paper only in the degenerate form (Schuler's par. Io, e.g.)

$$
\frac{d^{2} \theta}{d t^{2}}=0
$$

* The force component of gravity, due to tilting locally of the platform and acting on the proof mass, is in magnitude $m g \sin \theta, \theta$ being here the tilt angle locally. It was discovered by Newton (loc. cit. supra) that the tangential 'restoring' force required for tautochronism in circumferential motion has the magnitude

$$
\frac{R \theta}{R \sin \theta} \cdot m g \sin \theta
$$

where $\theta$ is here the indicated circumferential position. The illusion of the computer is that these $\theta$ 's are indistinguishable. The computer is, and must be, so designed that the tangential restoring force has the magnitude

or $(\theta \neq 0)$

$$
\frac{R \theta}{R \sin \theta} \cdot m g \sin \theta
$$

$$
\frac{m g}{R}(R \theta)
$$

whether $\theta$ is the local rotation or the circumferential position. For 'the force required to constrain a proof-mass carried in the vehicle to move with it' must also always be in balance with the force of inertia of the proof mass, by d'Alembert's principle, and is, indeed, 'le force perdu' of d'Alembert ( $174^{2}$ ). It is interesting that Newton must have understood and used d'Alembert's principle in making the above-mentioned discovery ( 1686 ). 
which has only the solutions $\dot{\theta}=$ constant, or $\theta=0$. It is easy to deduce from his equations, e.g.

$$
\frac{\Theta}{m g a}=\frac{R}{g}
$$

(Schuler's eq. 5)

and

$$
\frac{J}{m g a \cdot u \cdot \cos \varphi}=\frac{R}{g}
$$

(Schuler's par. $3^{1}$ )

by simply multiplying both members by $g$, that his apparatus is independent of $g$ !一 and hence, again, fundamentally different from that characterized by equations (1) and (2), above. In inertial navigation, it is Earth that is in tune, and there is no possibility of altering the period by tinkering with the device. I do not think that anyone can produce an inertial navigator with any other period, as, e.g., the period of 'about thirty minutes' reported by Schuler in his 1923 paper, par. 31 , as his best approach to an apparatus 'with full 84 -minute period.'

\section{The Schuler Pendulum and Inertial Navigation}

\section{Professor A. Stratton}

F. C. Bell in his contribution raises most interesting philosophical questions. In commenting on them, I will refer to a recent translation (Navigation U.S.A., 14, 26) of Schuler's paper and use the same notation.

Gravity and the Schuler pendulum

The impossibility of distinguishing by any physical measurement between a gravitational force and the inertial reaction force of an accelerating frame of reference is fundamental. In Fig. I our observer in an enclosed laboratory is observing the compression of a linear spring supporting a 'proof mass' $m$ (he knows the natural uncompressed

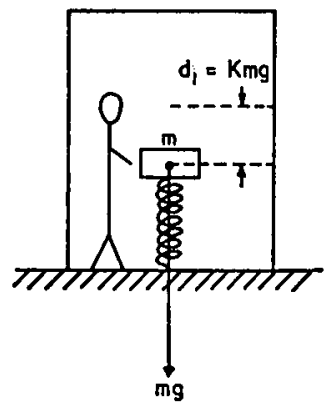

(a)

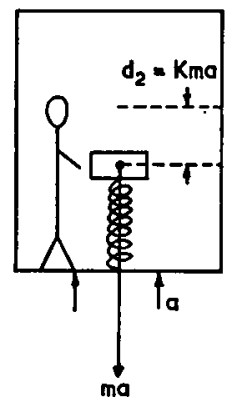

(b)
FIG I (a). Gravitation (b) Inertia length of the spring). In situation I(a) the laboratory-unknown to him-is sitting on the surface of the Earth and the spring is compressed to $d_{1}=k m g$ by the gravitational force of $g$ per unit mass acting on $m$ ( $k$ is a constant of the spring). In situation $\mathrm{I}(\mathrm{b})$ the laboratory-also unknown to himis in free motion in space accelerating under the action of a silent and vibration-free propulsive motor generating a thrust of, $a$, per unit mass of the labora- 